\title{
Reflets
}

Revue ontaroise d'intervention sociale et communautaire

\section{Développement d'un programme bénévole pour les personnes psychiatrisées en crise}

\section{Micheline Lemieux}

Volume 6, numéro 1, printemps 2000

Approches d'intervention : définir et renouveler nos pratiques

URI : https://id.erudit.org/iderudit/026304ar

DOI : https://doi.org/10.7202/026304ar

Aller au sommaire du numéro

Éditeur(s)

Reflets : Revue ontaroise d'intervention sociale et communautaire

ISSN

1203-4576 (imprimé)

1712-8498 (numérique)

Découvrir la revue

Citer cet article

Lemieux, M. (2000). Développement d’un programme bénévole pour les personnes psychiatrisées en crise. Reflets, 6(1), 208-215.

https://doi.org/10.7202/026304ar

Tous droits réservés (C) Reflets : Revue ontaroise d'intervention sociale et communautaire, 2000
Ce document est protégé par la loi sur le droit d'auteur. L'utilisation des services d'Érudit (y compris la reproduction) est assujettie à sa politique d'utilisation que vous pouvez consulter en ligne.

https://apropos.erudit.org/fr/usagers/politique-dutilisation/ 


\title{
Développement d'un programme bénévole pour les personnes psychiatrisées en crise
}

\author{
Micheline Lemieux,
} MSS, Hearst

\section{Introduction}

C'est en intervenant comme conseillère en santé mentale auprès des gens qui traversent des crises personnelles et situationnelles que j'ai été intriguée par la situation des personnes qui souffrent d'un handicap psychiatrique de longue durée et qui traversent des crises psychiatriques. J'ai donc décidé de profiter de mon stage de maîtrise en service social à l'Université Laurentienne pour approfondir mes connaissances de ces réalités et des besoins de ces personnes. À cette occasion, j'ai élaboré un programme destiné à ces personnes qui s'appuierait sur la présence de bénévoles pouvant leur venir en aide et tentant d'améliorer leur fonctionnement et la qualité de leur vie. Ce programme sera offert par les Services de Counselling de Hearst, Kapuskasing et Smooth Rock Falls.

Le programme a pour objectif principal d'élargir le réseau de soutien des personnes psychiatrisées en crise et, ainsi, de les aider à demeurer dans leur milieu naturel et d'éviter le recours à l'hospitalisation, sauf en cas de nécessité clinique. 


\section{Au départ...}

On sait que l'intervention de crise faite par les professionnels est d'une grande importance. Pour être efficace, elle doit être rapide et structurée, d'où l'importance de posséder une solide formation théorique et pratique. Par ailleurs, on se questionne sur l'utilisation, l'efficacité et l'impact de l'aide naturelle, tout particulièrement celle de la famille, lors de périodes de crise, moments où les personnes psychiatrisées en ont le plus besoin (Aguillera 1994; Sullivan-Everstine et Everstine 1993). Différentes recherches montrent que les personnes qui sont affectées par un handicap psychiatrique de longue durée évoluent habituellement dans un petit réseau social et que celui-ci est souvent peu efficace pour leur venir en aide lorsqu'ils vivent une crise (Guay 1991; Harris et Bergman 1991). Cette difficile situation les amènera alors à préférer l'hospitalisation au maintien en milieu naturel.

Comme intervenante en santé mentale, je me suis donc posée la question suivante. Si les intervenantes et les intervenants aident l'individu et que celui-ci dispose d'un réseau élargi de soutien, la personne psychiatrisée serait-elle plus apte à traverser la période de crise tout en évitant une hospitalisation psychiatrique?

À cause du nombre élevé de clients, les professionnels de la santé mentale sont de moins en moins disponibles pour faire de l'accompagnement en milieu naturel. Cette lacune a un impact sur la durée et la sévérité de la crise psychiatrique. De plus, le retour de l'individu dans son milieu naturel, suite à une hospitalisation psychiatrique, est vécu difficilement lorsque son réseau de soutien est restreint, voire inexistant. Finalement, on note qu'après une crise, la personne a tendance à s'isoler davantage. Ainsi, une présence plus amicale et normalisante peut aider à diminuer certains symptômes d'anxiété et de stress présent lors de la période de crise.

Pour relever ces défis, on propose donc qu'après l'intervention des professionnels dans la situation de crise elle-même, ces derniers soient appelés à jouer un nouveau rôle en s'intégrant et en 
collaborant avec une équipe de bénévoles afin d'atténuer les effets de la crise chez la personne affectée par un handicap psychiatrique. Nous pensons qu'avec un meilleur soutien de la communauté, nous arriverons à diminuer les hospitalisations psychiatriques, à maintenir les personnes dans la communauté et ainsi à augmenter la qualité de vie des personnes qui sont affectées par un handicap psychiatrique de longue durée. Certes, on ne nie pas le fait que dans certains cas, l'hospitalisation puisse être nécessaire, voire inévitable. Par contre, il est possible de croire que ces personnes seraient probablement mieux en mesure de traverser les périodes de crise, tout en diminuant le recours aux services hospitaliers, si elles pouvaient bénéficier du soutien d'un réseau élargi.

\section{Définition de la crise psychiatrique}

Précisons d'abord que le handicap psychiatrique correspond à la maladie mentale qui affecte sévèrement et à long terme le fonctionnement général de la personne (p. ex. schizophrénie ou trouble bipolaire, etc.). Une période de crise survient lorsque la personne qui souffre d'un handicap psychiatrique se retrouve en déséquilibre au niveau psychologique. La crise ou période de désorganisation est celle où la personne n'arrive plus à prendre soin d'elle convenablement ou à prendre de bonnes décisions. Dans ces cas, les symptômes les plus sévères que nous trouvons chez ces personnes sont les délires, la paranoïa, les hallucinations auditives et visuelles, la manie, les idées suicidaires, la confusion et la dépression (Slaikeu 1990). On trouve aussi d'autres symptômes moins sévères, mais aussi importants comme un laisser-aller dans l'hygiène personnelle, la désorientation, le discours incohérent, le manque de concentration, les sautes d'humeurs, l'isolement, etc. et l'incapacité de demander les services pouvant répondre à ses besoins. Certains symptômes seront plus faciles à évaluer, tandis que d'autres nécessiteront une plus grande période d'observation. Par ailleurs, la crise peut avoir diverses sources dont le développement de la maladie mentale, une période de 
décompensation suite à une période de stabilité ou encore, un changement important dans la vie de la personne telle un décès, un déménagement, un changement financier, etc. La durée et l'intensité des symptômes varieront selon la situation de la personne et l'aide accessible qui lui est offerte.

\section{Regards sur les besoins d'accompagnement des personnes qui souffrent d'un handicap psychiatrique}

Les gens qui souffrent d'un handicap psychiatrique de longue durée ont des besoins fondamentaux, comme tout autre individu, en plus d'avoir certains besoins plus spécifiques à leur état de santé. En s'inspirant de Lecomte (1991a;1991b) et des recherches de Santé Canada, faites en collaboration avec la Société canadienne de schizophrénie (1991), on peut diviser leurs besoins en cinq catégories : économiques, résidentiels, interpersonnels, thérapeutiques et temporels.

Les besoins économiques sont habituellement les plus importants. C'est grâce aux ressources financières que les gens réussissent à combler plusieurs besoins essentiels. On sait que la situation financière est considérée comme une des plus importantes sur l'échelle du stress et ce, même pour les gens qui sont affectés par un handicap psychiatrique. Il est donc important pour eux d'avoir une certaine stabilité financière afin de répondre à certains besoins essentiels tels la nourriture, le logement, etc. Parmi ces besoins, le transport est crucial, car ces personnes ont souvent à se déplacer pour divers motifs comme rendre visite à leur médecin, aller à la pharmacie ou plus simplement, parer aux exigences de la vie quotidienne dans la communauté. La personne affectée par un handicap psychiatrique éprouve souvent de la difficulté à faire un budget et doit être accompagnée d'une autre personne pour le gérer efficacement (p. ex. payer les factures, aller à la banque, etc.). 
Les besoins résidentiels sont fondamentaux. Ici, l'accompagnement vise à s'assurer, par exemple, que la personne habite dans un environnement sécuritaire et sans violence, dans un logement abordable et conforme aux normes de salubrité publique.Vivre dans un lieu d'habitation sain et acceptable, à l'extérieur de l'hôpital, est nécessaire au rétablissement à long terme de la personne qui souffre d'un handicap psychiatrique.

Les besoins interpersonnels surviennent à la suite de l'identification des symptômes négatifs de certaines maladies mentales comme la schizophrénie. Les soutiens familial et social deviennent essentiels pour aider la personne à réintégrer la communauté et à briser l'isolement social. Ceux et celles qui fournissent le soutien peuvent aider la personne psychiatrisée à réaliser ses plans à court terme, à entretenir son foyer et à s'occuper de son hygiène personnelle et ainsi, à augmenter ses habiletés de fonctionnement dans la vie quotidienne. La personne qui souffre d'un handicap psychiatrique a aussi besoin d'aide pour remplir des documents ou pour être orientée vers les services appropriés. L'accompagnement instrumental devient ainsi une aide que les proches peuvent procurer.

Les besoins thérapeutiques consistent à établir le suivi médical (p. ex. rendez-vous chez le médecin ou à la pharmacie, etc.). La personne affectée par un handicap psychiatrique a besoin d'être accompagnée quand elle passe de la phase aiguë d'une crise à celle de la réadaptation. Après avoir quitté l'hôpital pour retourner vivre dans son milieu, l'individu nécessite de l'accompagnement et du soutien émotionnel. L'accompagnement est essentiel lors de la période de réadaptation qui a pour but de passer de la dépendance à l'autonomie. À cette étape, on trouve aussi le besoin d'acquérir des habiletés de vie et le besoin d'intégration communautaire.

Les besoins temporels évoquent l'idée de se donner un emploi du temps. On parle ici des besoins de s'actualiser, de se réaliser à travers les loisirs et toutes autres activités sociales. L'accompagnement à travers ce processus favorisera l'augmentation de l'estime de soi et de la confiance en soi. Ce sont aussi des besoins importants qui évitent à la personne de sombrer dans la solitude et l'isolement. 


\section{Le but du programme d'accompagnement}

Plus le réseau social est petit, plus vite il s'épuise. Il s'avère donc souvent peu efficace pour apporter un soutien et pour répondre aux besoins de la personne lors d'une période de crise. Celle-ci étant très difficile à traverser, la personne sera souvent amenée à préférer l'hospitalisation, tout particulièrement si son réseau social est pauvre. Dans de telles circonstances, l'hospitalisation peut procurer au client en crise la stabilité et la sécurité dont il a besoin. C'est pour pallier les manques d'une telle situation qu'on a songé à mettre sur pied un nouveau programme. Le but de ce programme est de renforcer le réseau social naturel, en augmentant le nombre des membres du réseau afin que ceux-ci partagent différentes tâches orientées vers les besoins de la personne en crise. Le fait de renforcer et d'élargir le réseau social naturel amène une meilleure intervention et plus de support lors des crises psychiatriques. Plus la personne se sent entourée et acceptée, mieux elle arrive à diminuer l'intensité et la durée de la crise. Toutefois, un réseau social inefficace, peu importe son nombre, ne peut pas aider à garder le client dans la communauté.Ainsi, un des défis importants du programme est de rendre le réseau le plus efficace possible. L'objectif principal de notre programme est d'offrir un support naturel et amical dans le milieu naturel de la personne.

Un second objectif est de diminuer le nombre d'hospitalisations psychiatriques. Nous croyons que si les gens qui souffrent d'un handicap psychiatrique ont moins recours à l'hospitalisation, meilleure sera leur qualité de vie. Nous savons que le retour dans le milieu naturel, suite à l'hospitalisation, est un processus long et difficile pour la personne. De plus, nous croyons que l'hospitalisation psychiatrique accentue la stigmatisation et les préjugés rattachés à la maladie mentale. Si la personne en crise pouvait continuer à fonctionner dans son milieu, on pourrait ainsi favoriser l'élimination de mythes liés à la santé mentale. Ce serait un pas de plus vers la sensibilisation et l'éducation sur les préjugés reliés à la maladie mentale. 
Un troisième objectif du programme est de diminuer la dépendance envers les intervenants professionnels, afin de favoriser l'autonomie de la personne en crise. Une présence naturelle contribue à accroître la confiance en soi et l'estime de soi de la personne qui souffre d'un handicap psychiatrique. On croit aussi que la présence des professionnels tend à accentuer, elle aussi, les préjugés relatifs à la maladie mentale.

\section{Comment faire?}

L'objectif de ce programme est de former une équipe de bénévoles qui viendra agrandir le réseau naturel de soutien. Suite à une formation appropriée, les bénévoles seront plus aptes et mieux outillés pour accompagner la personne en crise, pour favoriser l'entraide entre les personnes impliquées dans le réseau et pour aider à la réinsertion sociale de la personne souffrant d'un handicap psychiatrique.

La formation de base des accompagnateurs bénévoles se divise en deux parties. Dans la première, on voit :

- l'historique, le mandat, les services et la clientèle de l'agence;

- le but du programme d'accompagnement;

- le rôle des intervenantes et des intervenants professionnels;

- le rôle des bénévoles;

- le processus de l'aide naturelle.

Dans la seconde partie de la formation, on se concentre sur :

- la démystification des maladies mentales;

- les différents types de maladies mentales (schizophrénie, trouble bipolaire, etc.);

- les traitements des maladies mentales;

- la définition de la crise psychiatrique et les besoins de la personne en crise;

- les tâches, les responsabilités, les avantages, le code de conduite des personnes bénévoles; 
- les situations difficiles (accidents, blessures, comportements incontrôlables, risque de suicide) et, finalement,

- l'encadrement des bénévoles offert par l'agence.

\section{Conclusion}

Ce stage m'a permis de mettre en place l'ensemble des conditions nécessaires à la réalisation de ce nouveau programme d'accompagnement des personnes psychiatrisées. Le programme sera offert par les Services de Counselling de Hearst, Kapuskasing et Smooth Rock Falls et constituera une composante du volet communautaire.Après une période de rodage, ce programme sera évalué et revu le cas échéant, afin de mieux répondre aux besoins des personnes psychiatrisées qui traversent une période de crise.

\section{Bibliographie}

AGUILlERA, D. (1994). Crisis Intervention:Theory and Methodology, California, Mosby.

GUAY,J. (1991). «L'approche proactive et l'intervention de crise», Santé mentale au Québec, vol. XVI, no 2,139-154.

HARRIS, M. et H. BERGMAN (1991). "Networking with Young Adults with Psychiatrics Disabilities", Psychiatric Rehabilitation, 191-202.

LECOMTE,Y. (1991a). «Les patients chroniques hospitalisés dans les départements de psychiatrie des hôpitaux généraux», Santé mentale au Québec, vol. 16, no 2, 9-12.

LECOMTE, Y. (1991b). «Les mécanismes d'adaptation des malades mentaux chroniques à la vie quotidienne», Santé mentale au Québec, vol. 16, no 2, 99-120.

SANTÉ CANADA, EN COLLABORATION AVEC LA SOCIÉTÉ CANADIENNE DE SCHIZOPHRÉNIE (s.d.). Redéfinir la schizophrénie : guide à l'intention des familles, Ottawa, Ministre des Approvisionnements et Services Canada.

SULLIVAN-EVERSTINE, D. et L.EVERSTINE (1993). Des gens en crise :l'intervention psychologique d'urgence, Marseille, Hommes et Perspectives.

SLAIKEU, K.A. 1990. Crisis Intervention. A Handbook for Practice and Research, Boston, Allyn and Bacon. 